\title{
Hypercalcemia associated with adenosquamous pancreatic carcinoma: A reason to initiate palliative treatment
}

\author{
Eudaldo M. López-Tomassetti-Fernández ${ }^{1}$, Julián Favre-Rizzo ${ }^{1}$, Luciano Delgado-Plasencia ${ }^{2}$ and Juan \\ Ramón Hernández-Hernández ${ }^{1}$
}

\begin{abstract}
${ }^{I}$ Department of Gastrointestinal Surgery. Hospital Universitario Insular de Gran Canaria. Las Palmas de Gran Canaria, Spain. ${ }^{2}$ Department of Gastrointestinal Surgery. Hospital Universitario de Canarias. La Laguna, Tenerife. Spain
\end{abstract}

\begin{abstract}
Background: hypercalcemia in patients with diagnosed carcinoma has predominantly a humoral basis mediated by parathyroid hormone-related protein (PTH-rP). Among the reported cases, hypercalcemia associated with the majority of abdominal malignancies indicates an advanced stage of disease.

Case report: we present a case of a 78-year-old patient with an adenosquamous pancreatic carcinoma associated with humoral hypercalcemia mediated by PTH-rP.

Conclusion: in this case, demonstration of unexpectantly rapid increase in calcium serum correlated with aggressive tumor growth led us to raise the hypothesis that PTH-rP could be a mediator of invasion and dissemination secreted by some tumors, and probably indicates the appropriate time to initiate palliative treatment.
\end{abstract}

Key words: Adenosquamous carcinoma. Pancreatic malignancy. Humoral hypercalcemia.

\section{INTRODUCTION}

Humoral hypercalcemia of malignancy mediated by parathyroid hormone-related protein (PTHrP) is an important paraneoplastic syndrome occurring in humans with a wide variety of cancers. When hypercalcemia is present, a bunch of symptoms may occur usually depending on the

López-Tomassetti-Fernández EM, Favre-Rizzo J, Delgado-Plasencia L, Hernández-Hernández J. Hypercalcemia associated with adenosquamous pancreatic carcinoma: A reason to initiate palliative treatment. Rev Esp Enferm Dig 2013;105:425-428.

Received: 07-02-2013

Accepted: $11-03-2013$

Correspondence: Julián Favre-Rizzo. Department of Surgery. Hospital Universitario Insular de Gran Canaria. Avda. Marítima del Sur, s/n. 35016 Las Palmas. Las Palmas de Gran Canaria, Spain

e-mail: julianfavre@ hotmail.com degree of hypercalcemia and the rapidity with which serum calcium concentration increases (1).

We present a case of a patient who was admitted to our service with cognitive impairment due to humoral hypercalcemia associated with abdominal malignancy. We discuss the overall prognosis of these patients and the best treatment of this condition based on a review of the literature.

\section{CASE REPORT}

A 78 years-old woman was admitted to our hospital with important cognitive impairment and behavioral disorders lasting two weeks. Her family also referred that at home she was easily lulled into a somnolent state and had a body weight loss of approximately $10 \mathrm{~kg}$ within the last month. Her past medical history was unremarkable but curiously she was admitted to our hospital 2 months before due to blunt abdominal traumatism with suspected spleno-pancreatic hematoma reason why she was being followed-up at our hospital.

At her new admission she was extremely somnolent with scarce collaboration on physical exam. It could be felt a non-painful mass in the epigastric region without other specific findings.

Routine laboratory workup revealed hematocrit: $37 \%$, hemoglobin: $12.4 \mathrm{~g} / \mathrm{dL}$, leukocytosis $\left(23,400 \mathrm{~mm}^{3}\right)$, prothrombin: $89 \%$ (normal $100 \%$ ), and the platelet count was $362,000 / \mathrm{mm}^{3}$.

Serum biochemistry showed glucose: $114 \mathrm{mg} / \mathrm{dL}$, hypercalcemia (calcium: $15.2 \mathrm{mg} / \mathrm{dL}$ ), lipase: $24 \mathrm{U} / \mathrm{L}$, amylase: $<30 \mathrm{U} / \mathrm{L}$, inorganic phosphorus: $3.6 \mathrm{mg} / \mathrm{dL}$, magnesium: $1.4 \mathrm{mg} / \mathrm{dL}$, total bilirrubin: $0.4 \mathrm{mg} / \mathrm{dL}$, alkaline phosphatase: $132 \mathrm{U} / \mathrm{L}$, ALT: $55 \mathrm{UI} / \mathrm{L}$; AST: $40 \mathrm{UI} / \mathrm{L}$, blood urea nitrogen: $28 \mathrm{mg} / \mathrm{dL}$ and creatinine: $1.8 \mathrm{mg} / \mathrm{dL}$.

A new CT-scan showed that the suspected hematoma had changed since the last control and the diagnosis of car- 
cinoma with a rapid tumor growth was suspected (Fig. 1). There was round enhancement of the suspected tumor on contrast $\mathrm{CT}$ and bad defined areas of necrosis.

MRI was considered to better delineate the process and it concluded that a $10 \mathrm{~cm}$ mass depending from the pancreatic tail was infiltrating the kidney, stomach and left adrenal gland.

New analysis revealed that carcinoembryonic antigen (CEA) was $3.9 \mathrm{ng} / \mathrm{mL}$ (normal, $<5.3 \mathrm{ng} / \mathrm{mL}$ ), carbohydrate antigen 19-9 (CA19-9) was $468.5 \mathrm{U} / \mathrm{mL}$ (normal, < $40 \mathrm{U} /$ $\mathrm{mL}$ ), CA 125 was 55.5, high-sensitivity PTH was 22.20 $\mathrm{pg} / \mathrm{mL}$ (normal range, $9.00-78.00 \mathrm{pg} / \mathrm{mL}$ ), PTH-rP was 9.4 $\mathrm{pmol} / \mathrm{L}$, (normal, $<0.6 \mathrm{pmol} / \mathrm{L}$ ), and calcitriol was $48 \mu \mathrm{g} /$ $\mathrm{mL}$ (normal range $18-78 \mu \mathrm{g} / \mathrm{mL}$ ).

CT-scan of the neck and chest was unremarkable and bone gammagraphy did not show any pathological activity, so the patient was treated with calcitonin, furosemide and bisphosphonates until serum calcium level fell to normal.

An operative intervention was scheduled due to rapid recovery of the patient. It showed a huge irresectable mass depending from the pancreatic tail firmly attached to the retroperitoneum and stomach with small size peritoneal deposits. A biopsy of the mass was performed and the laparotomy was closed. Histopathological study of the specimen showed a poor differentiate adenosquamous carcinoma (Figs. 2 and 3). The postoperative course was uneventful and the patient was discharged for palliative care. No systemic chemotherapy or radiotherapy was given because of the patient's poor prognosis.

The patient died 3 months later because of widespread tumor progression. Two weeks before her death she proved to have optimal cognitive status and calcium levels with
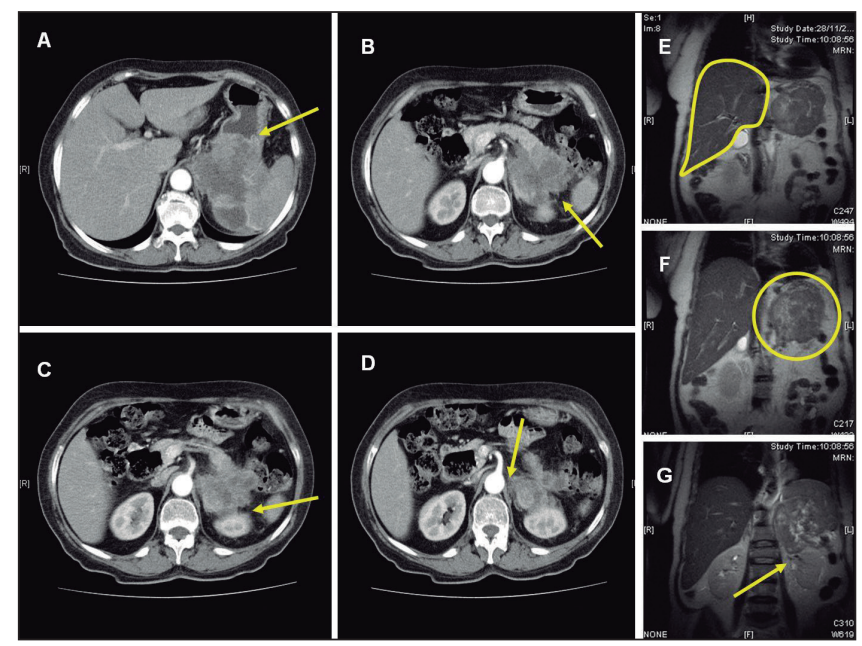

Fig. 1. Preoperative study of the patient. A. CT-scan showed a big pancreatic tumor infiltrating the stomach. B. It can clearly be seen that the tumor depends from the pancreatic tail and it is invading the left adrenal gland. C. The tumor is also extending and invading the left kidney. D. Extensive infiltration of the retroperitoneum and close relation to the aorta. E. The liver is free of disease. F. Tumor mass on MRI (coronal plane). G. Huge tumor invading the left kidney and adrenal gland on MRI.
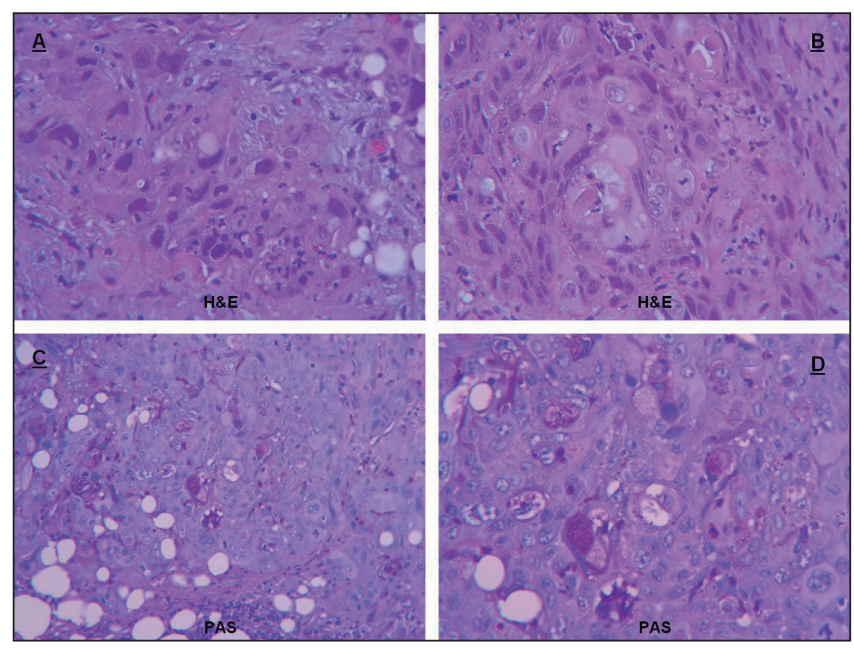

Fig. 2. A, B. High-power hematoxylin-and-eosin stain shows multiple squamous epithelial and mucosecretory cells. C, D. Periodic Acid-Schiff stain (PAS) confirmed the presence of mucous secretion.

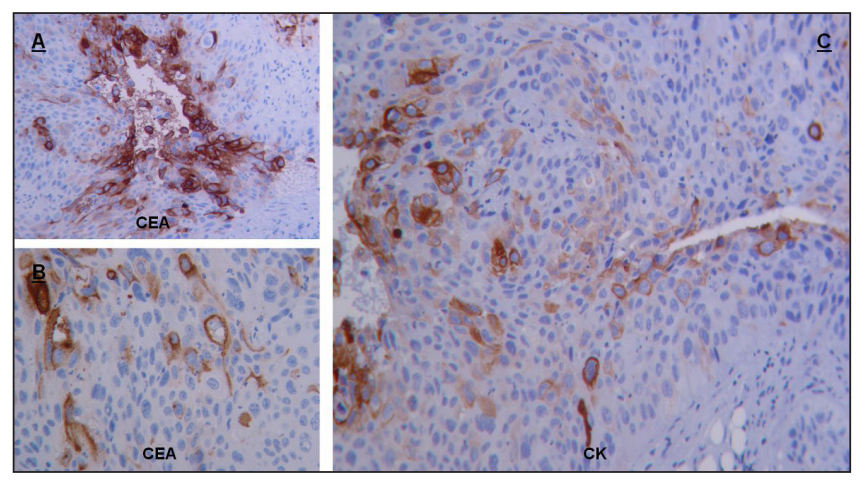

Fig. 3. A, B. Immunohistochemical stain shows positive tissue staining for CEA. C. Immunohistochemical stain showed the tumor cells to be positive in cytokeratin.

medical treatment based on bisphosphonates and adequate hydration.

\section{DISCUSSION}

Hypercalcemia is not an infrequent metabolic disorder in patients with neoplastic disease $(2,3)$. The incidence of hypercalcemia of malignancy ranges from 3 to $35 \%$ (2) depending on the origin of the tumor and its particular histology. Among patients with solid tumors and hypercalcemia, at least $80 \%$ have increased serum concentrations of PTH-rP. Thus, hypercalcemia in patients with cancer has predominantly a humoral basis, rather than being caused by local destruction of bone by tumor metastases.

Most pancreatic tumors originate in the duct cells or in the cells that produce digestive enzymes; so called exocrine pancreatic cancer. The most frequent is ductal adenocarcinoma of the pancreatic head. Adenosquamous carcinoma of the pancreas is a rare variant $(4,5)$ that is 
generally diagnosed at an advanced stage of disease and therefore has a poor prognosis $(4,6)$.

Malignant hypercalcemia associated with an exocrine pancreatic malignancy is not a new concept but has been rarely reported $(7,8)$. It has been reported in association with local osteolytic effect of the bones (local hypercalcemia) and secondary to some tumor-derived compounds (humoral hypercalcemia) such as: PTH-rP, vitamin D3 and some cytokines (7). This latter form is particularly frequent in endocrine pancreatic cancer but extremely rare in exocrine pancreatic carcinoma. In our case, severe hypercalcemia with normal plasma concentrations of PTH and vitamin D3 was compatible with humoral malignant hypercalcemia secondary to undifferentiated adenosquamous carcinoma once bone metastases were excluded. High levels of the tumor mediator PTH-rP (10.3 pmol/L; normal, $<0.6 \mathrm{pmol} / \mathrm{L}$ ) seems to be involved in the development of hypercalcemia.

Recently, Kakizaki (8) reported only 15 cases of exocrine pancreatic cancer associated with humoral hypercalcemia including his case until 1998. In only 6 cases hypercalcemia was caused by PTH-rP and in none of them had the adenosquamous histology ( 2 cases). Few cases of exocrine pancreatic cancer with malignant hypercalcemia have been reported in the English literature since Kakizaki's (8) review.

To our knowledge, only two cases of adenosquamous pancreatic cancer associated with humoral hypercalcemia in which PTH-rP was identified as the causative factor have been properly reported before $(9,10)$. Our case represents the third in the literature. Remarkably the case reported by Inoue and ours showed similar findings: rapid tumor growth in close association with increased calcium levels; in spite that our patient had leukocytosis and severe cachexia at the time of diagnosis. Importantly, in all reported cases (8-10), hypercalcemia represented an advanced stage of disease and appropriate time to initiate palliative treatment. However, in our case we decided to explore the abdominal cavity because the condition of the patient had greatly improved since admission and the diagnosis was not totally clear. Laparotomy confirmed that disease was systemic and locally unresectable.

The combination of intravenous saline, furosemide, bisphosphonates and calcitonin was very effective in this case. Many case reports and series have shown that bisphosphonate therapy can successfully control hypercalcemia due to excess PTH-rP secretion during the very short life expectancy of these patients. However, in some cases hypercalcemia can recur. These cases have a dismal prognosis heralding imminent death $(9,11,12)$. In our case, medical treatment proved effective. It allowed early ambulation improving the quality of life of the patient with no adverse effects.

Interestingly, in the present case our patient was being followed for a suspected spleno-pancreatic hematoma that was an aggressive tumor. Thus, we could demonstrate com- paring both CT-scans and serum calcium that calcium levels were unexpectantly increased during tumor growth and invasion. In only two months the serum calcium increased from $9.4 \mathrm{mg} / \mathrm{dL}$ to $15.2 \mathrm{mg} / \mathrm{dL}$ while the mass showed a rare growth pattern with distinctly malignant behavior based on CT-scan and MRI.

Recently some investigators (13) have suggested that local bone-derived factors further enhance the growth potential of some of these tumors, resulting in cycles of bone resorption leading to further tumor growth leading to more bone resorption. In fact, the present case suggests the same principle. Therefore, we hypothesized that there could be a threshold limit in some adenosquamous carcinoma where the amount of PTH-rP secreted by the tumor promotes invasion and dissemination. This obviously represents an advance stage of disease.

Both mechanisms suggest that bone-derived factors or high calcium concentrations could be necessary during invasion and dissemination; and for this purpose, some products such as PTH-rP and other different unknown mediators are secreted. This similar finding has led some authors to consider hypercalcemia and PTH-rP as potential markers of systemic disease in pancreatic adenocarcinoma (14).

If this hypothesis is finally proved, new drugs either limiting calcium consumption by tumoral cells or neutralizing PTHrP could be design $(15,16)$. In normal conditions, $\mathrm{PTH}-\mathrm{rP}$ is secreted to regulate local tissue functions with diverse biologic roles (central nervous system, effects on skin, cartilages, placenta...). However, PTH-rP might have other unknown functions yet. For example, recent research has shown that PTH-rP induces tumoral cachexia in experimental animals and new monoclonal antibodies which neutralize PTHrP have shown promising results against these undesirable effects.

In conclusion, high PTH-rP levels seem to be a good marker of advanced disease in some types of pancreatic cancer. Therefore, in those cases where PTH-rP is found to be high, most sensitive exams (PET, CT-scan, MRI) should be justified as a preoperative test to rule out systemic disease. Moreover, surgeons should strongly consider diagnostic laparoscopy in order to avoid an unnecessary laparotomy. This review suggests that high PTH-rP levels and hypercalcemia usually is the proper time to initiate palliative treatment.

\section{REFERENCES}

1. Inzucchi SE. Understanding hypercalcemia. Its metabolic basis, signs, and symptoms. Postgrad Med 2004;115:69-70.

2. Pecherstorfer M, Schilling T, Blind E, Zimmer-Roth I, Baumgartner G, Ziegler R, et al. Parathyroid hormone-related protein and life expectancy in hypercalcemic cancer patients. J Clin Endocrinol Metab 1994;78:1268-70.

3. Pecherstorfer M, Brenner K, Zojer N. Current management strategies for hypercalcemia. Treat Endocrinol 2003;2:273-92.

4. Rahemtullah A, Misdraji J, Pitman MB. Adenosquamous carcinoma of the pancreas: Cytologic features in 14 cases. Cancer 2003;99:372-8. 
5. Kardon DE, Thompson LD, Przygodzki RM, Heffess CS. Adenosquamous carcinoma of the pancreas: A clinicopathologic series of 25 cases. Mod Pathol 2001;14:443-51.

6. Hsu JT, Yeh CN, Chen YR, Chen HM, Hwang TL, Jan YY, et al. Adenosquamous carcinoma of the pancreas. Digestion 2005;72:104-8.

7. Esbrit P. Hypercalcemia of malignancy -new insights into an old syndrome. Clin Lab 2001;47:67-71.

8. Kakizaki S, Ohya N, Yoshinaga T, Higuchi T, Kitazawa R, Takayama $\mathrm{H}$, et al. Undifferentiated pancreatic cancer associated with humoral hypercalcemia of malignancy. Jpn J Clin Oncol 1998;28:563-6.

9. Inoue T, Nagao S, Tajima H, Okudaira K, Hashiguchi K, Miyazaki J, et al. Adenosquamous pancreatic cancer producing parathyroid hormone-related protein. J Gastroenterol 2004;39:176-80.

10. Hyodo N, Tashiro T, Hyodo T, Yamanaka T, Ota M, Yamada S. A case of adenosquamous pancreatic cancer with high serum level of parathyroid related protein in which both primary and liver metastatic lesions showed remarkable cystic changes. Nippon Shokakibyo Gakkai Zasshi (Japanese Journal of Gastroenterology) 1999;96:181-8.
11. Ralston SH, Gallacher SJ, Patel U, Campbell J, Boyle IT. Cancer-associated hypercalcemia: Morbidity and mortality. Clinical experience in 126 treated patients. Ann Intern Med 1990;112:499-504.

12. Stewart AF. Clinical practice. Hypercalcemia associated with cancer N Engl J Med 2005;352:373-9.

13. Goltzman D, Karaplis AC, Kremer R, Rabbani SA. Molecular basis of the spectrum of skeletal complications of neoplasia. Cancer 2000;88:2903-8.

14. Rasnake MS, Glanton C, Ornstein D, Osswald M, Garrison M. Hypercalcemia mediated by parathyroid hormone-related protein as an early manifestation of pancreatic adenocarcinoma metastasis: A case report. Am J Clin Oncol 2001;24:416-7.

15. Sato K, Onuma E, Yocum RC, Ogata E. Treatment of malignancy-associated hypercalcemia and cachexia with humanized anti-parathyroid hormone-related protein antibody. Semin Oncol 2003;30:167-73.

16. Iguchi H, Aramaki Y, Maruta S, Takiguchi S. Effects of anti-parathyroid hormone-related protein monoclonal antibody and osteoprotegerin on PTHrP-producing tumor-induced cachexia in nude mice. J Bone Miner Metab 2006;24:16-9. 